\title{
Electrodeposited Ni/Ge Contacts for Limiting Leakage Currents in Schottky Barrier MOSFETs
}

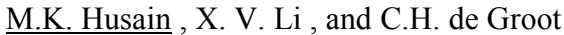

School of Electronics and Computer Science, University of Southampton, Southampton, UK

Ge channel MOSFETs are considered as promising devices for high-speed CMOS technology as they offer high carrier mobility suitable for large drive current. A Ge channel Schottky barrier (SB)-pMOSFET, however, suffers from large leakage currents due to its narrow bandgap and low SB height [1]. In a recent work by the authors it was shown that SBs prepared on $\mathrm{Si}$ by electrodeposition exhibit superior properties to those prepared by physical vapor deposition [2]. In the present work, we show that electrodeposited $\mathrm{Ni} / \mathrm{Ge}$ and $\mathrm{NiGe} / \mathrm{Ge} \mathrm{SBs}$ formed even on highly n-doped Ge exhibit near ideal SB behavior with very low reverse leakage current. In order to suppress source-to-drain leakage currents in short channel SB-pMOSFETs, a highly n-doped substrate should be used. The low off-current exhibited by electrodeposited SBs on highly doped Ge might make this possible limiting the junction leakage current at the drain/body contact.

Typical current density $(J)$ versus applied voltage $(V)$ characteristics of electrodeposited $\mathrm{Ni} / \mathrm{Ge}$ SBs for various Ge resistivity $(\rho 1=2-2.4 \Omega$-cm, $\rho 2=0.13-0.15 \Omega$-cm and $\rho 3=0.005-0.02 \Omega$ $\mathrm{cm}$ ) are presented in Fig. 1. The SBs show rectification of up to five orders of magnitude. This is a significant achievement since SBs grown by evaporation technique exhibited rectification of only $2-3$ orders even on highly resistive Ge [3],[4]. The reverse leakage current in our SBs at 1-V bias is lower by at least an order of magnitude [4]. By fitting the thermionic emission model in the low forward bias region of the $J-V$ curves the barrier height $\Phi_{n}$, ideality factor $\eta$ and series resistance $\mathrm{R}_{\mathrm{s}}$ were extracted. Capacitance-voltage $(C-V)$ measurements on the SBs were also performed to extract $\Phi_{n}$ and doping density $\mathrm{N}_{\mathrm{d}}$. The SB parameters extracted by both methods are presented in Table I.

The SBs were annealed in an inert chamber at temperature range $300-500{ }^{\circ} \mathrm{C}$. The Ni film $(70 \mathrm{~nm})$ completely transformed into $\mathrm{NiGe}$ (shown in Fig. 2) at $500{ }^{\circ} \mathrm{C}$, which was confirmed by XRD analysis. $I-V$ curves of a $\mathrm{Ni}(70 \mathrm{~nm}) / \mathrm{Ge} \mathrm{SB}$ under various annealing conditions are presented in Fig. 3. The extracted barrier heights and ideality factors of the SBs under various annealing conditions are in the ranges of $0.52-0.54 \mathrm{eV}$ and $1.1-1.2$, respectively. This refers to the Fermi-level pinning in Ge and its thermal stability. The increase in high forward bias current with increasing annealing temperature indicates to the lower specific resistance of NiGe.

By using the TCAD simulator Sentaurus Device, the leakage current behavior of a $30 \mathrm{~nm}$ Ge channel SB-pMOSFET is predicted. The simulator was at first calibrated by using the Schottky barrier heights and doping density obtained by C-V method. The obtained J-V curves are in good agreement with the experimental characteristics as shown in Fig.4. The transfer characteristics of the simulated SB-pMOSFET are presented in Fig.5. It is observed that the subthreshold leakage current decreases when Ge doping is increased. This occurs at no additional cost of drain/body leakage due to the extremely low reverse bias currents in the electrodeposited $\mathrm{Ni} / \mathrm{Ge}$ Schottky diodes. Therefore, we propose that electrodeposition could be used for sourcedrain formation of a highly doped Ge-based SB-pMOSFET to achieve low subthreshold leakage current.

\section{References}

1. J. Larson and J. Snyder, "Overview and status of metal S/D Schottky barrier MOSFET technology," IEEE Trans. Electron Devices, vol. 53, no. 5, pp. 1048-1058, May 2006.

2. M. Kiziroglou, X. Li, A. Zhukov, P. de Groot, and C. de Groot, "Thermionic field emission at electrodeposited NiSi Schottky barriers," Solid State Electron., vol. 52, no. 7, pp. 1032-1038, Jul. 2008.

3. K. Ikeda, T. Maeda, and S. Takagi, "Characterization of platinum germanide/Ge(100) Schottky barrier height for Ge channel metal source/drain MOSFET," Thin Solid Films, vol. 508, no. 1/2, pp. 359-362, Jun. 2006.

4. S. Zhu and A. Nakajima, "Annealing temperature dependence on nickel-germanium solid-state reaction," Jpn. J. Appl. Phys., vol. 44, no. 24, pp. L753-L755, 2005. 


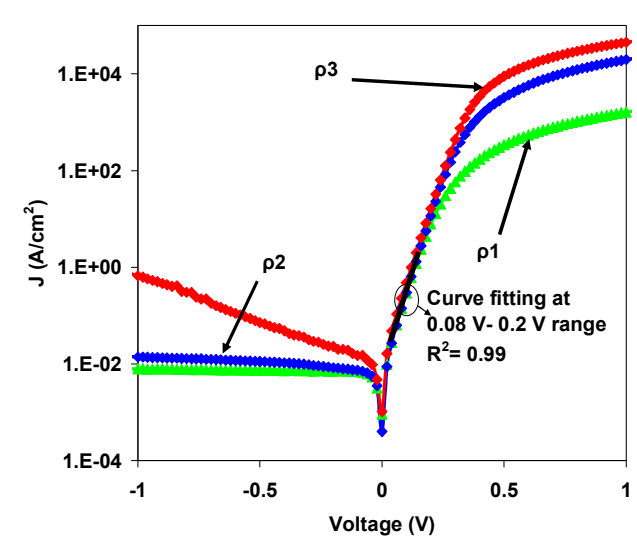

Fig. 1: $J-V$ characteristics of the Ni/Ge Schottky diodes (10 $\mu \mathrm{m}$ square contact) as a function of $\mathrm{Ge}$ resistivity.

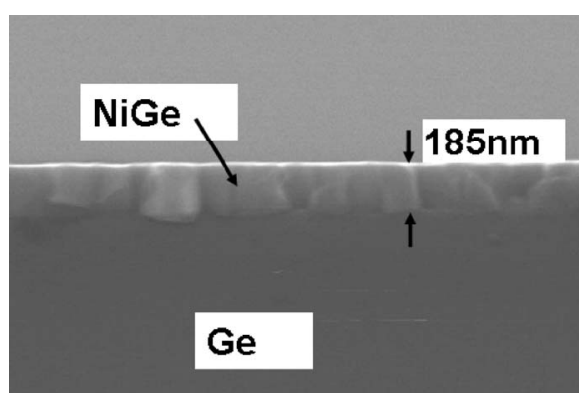

Fig. 2: Cross-sectional SEM image of a Ni/Ge sample annealed at $500{ }^{\circ} \mathrm{C}$.

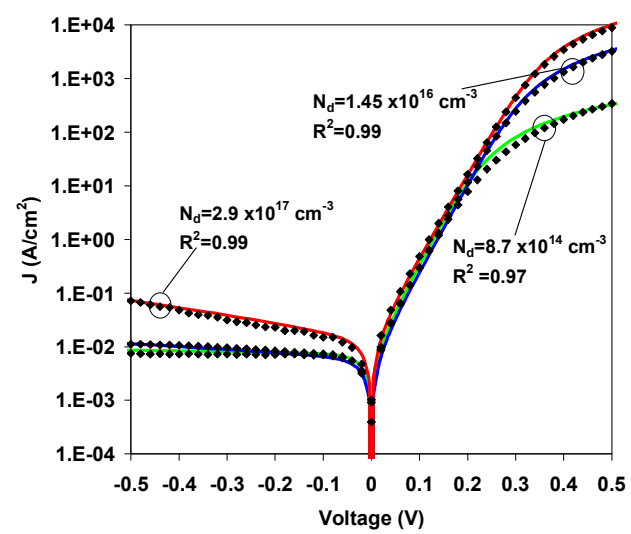

Fig. 4: $J-V$ characteristics (solid lines) for $10-\mu \mathrm{m}$ square $\mathrm{Ni} / \mathrm{Ge}$ contacts for various doping density modeled by Sentaurus Device simulator tool. The corresponding experimental curves (symbols) are also presented for reference.

\begin{tabular}{|c|c|c|c|}
\hline $\begin{array}{c}\text { Resistivity Label } \\
\rightarrow\end{array}$ & $\rho 1$ & $\rho 2$ & $\rho 3$ \\
\hline$\eta$ & 1.12 & 1.04 & 1.09 \\
\hline $\mathrm{R}_{s}(\Omega)$ & 30.77 & 16.05 & 11.72 \\
\hline $\begin{array}{c}\Phi_{n}(\mathrm{eV}) \\
(\text { from J-V) }\end{array}$ & 0.52 & 0.53 & 0.52 \\
\hline $\begin{array}{c}\Phi_{n}(\mathrm{eV}) \\
(\text { from C-V) }\end{array}$ & 0.56 & 0.53 & 0.53 \\
\hline $\mathrm{N}_{d}\left(\mathrm{~cm}^{-3}\right)$ & $8.7 \times 10^{14}$ & $1.45 \times 10^{16}$ & $2.9 \times 10^{17}$ \\
\hline $\begin{array}{c}\text { Resistivity }(\Omega- \\
\text { cm) } \\
\text { (nominal) }\end{array}$ & $2-2.4$ & $0.13-0.15$ & $0.005-$ \\
\hline $\begin{array}{c}\text { Resistivity }(\Omega- \\
\text { cm) } \\
\text { (from C-V) }\end{array}$ & 1.84 & 0.11 & 0.006 \\
\hline
\end{tabular}

Table I: Extracted $\Phi_{n}, \eta, \mathrm{R}_{s}(J-V$ method $)$ and $\Phi_{n}, \mathrm{~N}_{d}$, substrate resistivity ( $C-V$ method) of the $\mathrm{Ni} / \mathrm{Ge}$ Schottky diodes.

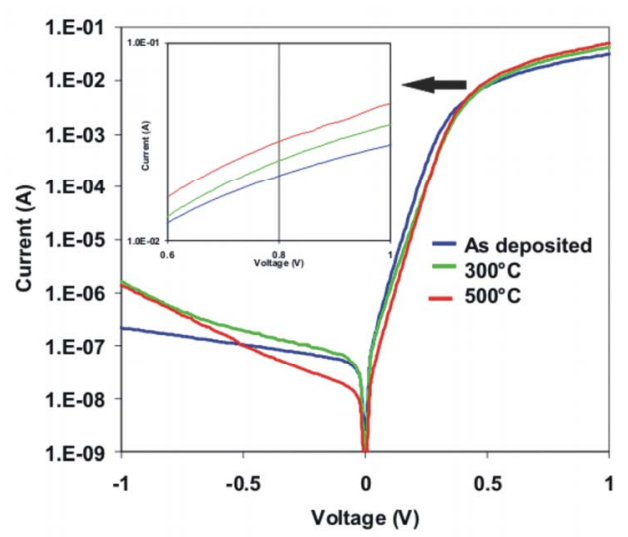

Fig. 3: $I-V$ characteristics of the $\mathrm{Ni} / \mathrm{Ge}(\rho 3)$ Schottky diodes (20 $\mu \mathrm{m}$ square), as a function of annealing temperatures.

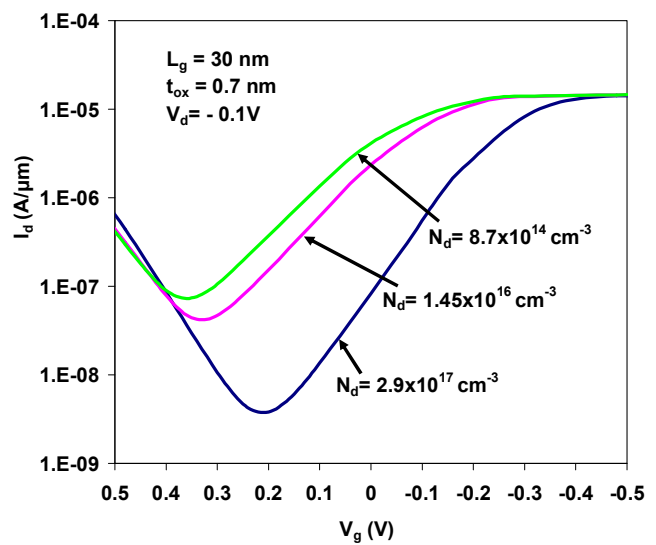

Fig. 5: Simulated transfer characteristics of 30nm Ge channel bulk SB-pMOSFET showing the effect of increasing substrate doping density on leakage currents. 